\title{
Cirurgia parendodôntica para remoção de um cisto periapical: relato de caso
}

\author{
Parendodontic surgery to remove a periapical cyst: case report \\ Cirugía endodóntica para remoción de quiste periapical: reporte de caso
}

\section{Resumo}

Objetivo: Descrever o caso clínico em que foi realizado uma cirurgia parendodôntica para remoção de um cisto periapical, associada à apicectomia e obturação retrógrada de MTA. Metodologia: Após assinatura do TCLE pelo paciente, realizou- o relato de um caso clínico analítico e descritivo realizado na Faculdade de Odontologia de Pernambuco, FOP - UPE. Resultados: Paciente de 19 anos de idade, gênero masculino, sem doenças de base, procurou o atendimento clínico na Faculdade de Odontologia de Pernambuco com o objetivo de finalizar o tratamento endodôntico em um dente portador de lesão periapical extensa e ápice aberto, uma vez que não se conseguiu obturar devido a drenagem de coleção purulenta via canal radicular. Após solicitação e análise da tomografia cone beam, optou-se pela execução de uma cirurgia paraendodôntica realizada para enucleação do cisto periapical e, após o corte do ápice, realizou-se a obturação retrógrada com MTA com o intuito de realizar em porta sessão a obturação do canal radicular. Considerações Finais: Constatou-se que a cirurgia parendodôntica aliada à apicectomia e obturação retrógrada com MTA determinou o sucesso do tratamento, resultando no reparo da radiotransparência óssea periapical.

Palavras-chave: Cisto radicular; Procedimentos cirúrgicos bucais; Endodontias.

\begin{abstract}
Objective: To describe a clinical case in which endodontic surgery was performed to remove a periapical cyst, associated with apicectomy and retrograde MTA obturation. Methodology: After signing the informed consent by the patient, an analytical and descriptive clinical case report was carried out at the Faculty of Dentistry of Pernambuco, FOP - UPE. Results: A 19-year-old male patient, without underlying diseases, sought clinical care at the Faculty of Dentistry of Pernambuco to with the aim of completing the endodontic treatment in a tooth with an extensive periapical lesion and open apex, a as the filling was not possible due to drainage of a purulent collection via the root
\end{abstract}


canal. After requesting and analyzing the, it was decided to perform a paraendodontic surgery performed for enucleation of the periapical cyst and, after cutting the apex, retrograde obturation with MTA was performed in order to perform the obturation in the next session. of the root canal. Final considerations: It was found that paraendodontic surgery combined with apicectomy and retrograde filling with MTA determined the success of the treatment, resulting in the repair of periapical bone radiotransparency.

Keywords: Radicular cyst; Oral surgical procedures; Endodontics.

\section{Resumen}

Objetivo: Describir un caso clínico en el que se realizó cirugía endodóntica para extirpación de un quiste periapical, asociado a apicectomía y obturación retrógrada de MTA. Metodología: Después de la firma del consentimiento informado por parte del paciente, se realizó un informe de caso clínico analítico y descriptivo en la Facultad de Odontología de Pernambuco, FOP - UPE. Resultados: Un paciente masculino de 19 años, sin enfermedades de base, buscó atención clínica en la Facultad de Odontología de Pernambuco para completar el tratamiento de endodoncia en un diente con lesión periapical extensa y ápice abierto, ya que no fue posible obturarse debido al drenaje de la colección purulenta a través del conducto radicular. Tras solicitar y analizar la tomografía de haz cónico, se decidió realizar una cirugía paraendodóntica realizada para la enucleación del quiste periapical y, tras el corte del ápice, se realizó una obturación retrógrada con MTA para realizar la obturación en sesión de puerta de la raíz. canal. Consideraciones Finales: Se encontró que la cirugía endodóntica combinada con apicectomía y obturación retrógrada con MTA determinaron el éxito del tratamiento, resultando en la reparación del hueso periapical radiolúcido.

Palabras clave: Quiste radicular; Procedimientos quirúrgicos orales; Endodoncia.

\section{Introdução}

O tratamento endodôntico conservador é a terapia de primeira escolha para a desinfecção do sistema de canais radiculares por meio preparo e uso de soluções irrigadoras. Entretanto, apesar da melhoria das técnicas e materiais utilizados na obturação do canal radicular, verifica-se ainda um alto índice de falha no procedimento convencional, com sucesso sido em torno de $65 \%$ a $90 \%$ dos casos (Pavelski et al., 2016).

Dessa forma, a cirurgia paraendodôntica revela-se alternativa para casos em que o tratamento e/ou retratamento endodôntico foram falhos, sendo indicada em casos de lesões periapicais persistentes, perfuração, fratura e degraus do terço apical, além de canais radiculares com acesso restrito via câmara pulpar devido a calcificações (Almeida-Filho et al., 2011; Oliveira et al., 2017; Graciano et al., 2021).

Como modalidades terapêuticas relacionadas à cirurgia parendodôntica, destacam-se a apicectomia e a obturação retrógrada com MTA. A apicectomia é a secção cirúrgica do segmento apical parcial de um dente, possibilitando a retrobturação do canal radicular a partir do ápice (Almeida-Filho et al., 2011; Silva \& Oliveira, 2016; Travassos et al., 2020). O material de escolha para o procedimento é o MTA, um cimento odontológico que oferece biocompatibilidade devido à liberação de íons hidroxila e formação de hidróxido de cálcio durante o processo de hidratação (Sousa et al., 2014; Pozza et al., 2005). Ademais, oferece correto preenchimento e vedação marginal, que impede o influxo de bactérias aos tecidos periapicais. Gera, então, melhor adaptação às paredes dentinárias durante a presa do material e induz, então, à regeneração tecidual (Pavelski et al., 2016; Oliveira et al., 2017).

\section{Metodologia}

Após assinatura do Termo de Consentimento Livre e Esclarecido (TCLE) pelo paciente, autorizando o uso de sua imagem para fins acadêmicos, o presente estudo visa relatar um caso clínico analítico e descritivo (Pereira et al., 2018), sobre uma cirurgia parendodôntica realizada na Faculdade de Odontologia de Pernambuco, FOP - UPE, com a realização de apicectomia e obturação retrógrada com MTA para remoção de cisto periapical.

\section{Resultados}

Paciente V.R.A.S. 19 anos de idade, gênero masculino, sem doenças de base, procurou o atendimento clínico na 
Faculdade de Odontologia de Pernambuco com o objetivo de finalizar o tratamento endodôntico em um dente portador de radiotransparência óssea periapical extensa, uma vez que o dentista iniciou a terapia não conseguia obturar o canal devido a drenagem de coleção purulenta via canal radicular.

Optou-se, então, por realizar a enucleação do cisto periapical através de uma cirurgia parendodôntica: apicectomia seguida da obturação retrógrada com MTA com intuito de realizar em outra sessão a obturação do canal radicular, a fim de se alcançar a reparação óssea periapical. Solicitou-se tomografia computadorizada de Cone Beam para avaliar o aspecto inicial do caso (Figura 1), pela vantagem de produzir imagens tridimensionais, possuir menor custo e menor dose de radiação (D’Azzio et al., 2010).

Figura 1: Tomografia de Feixe Cônico Cone Beam.

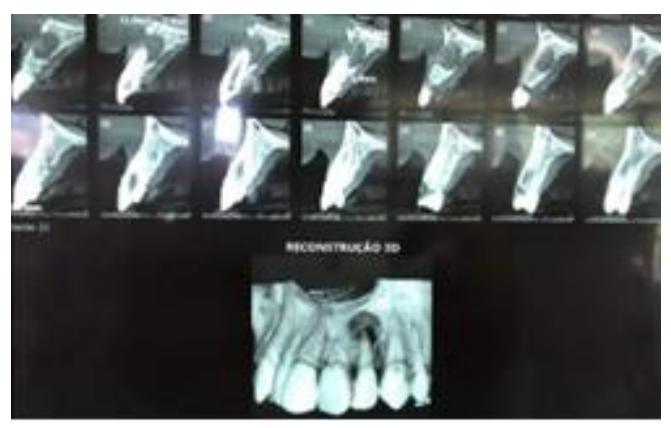

Fonte: Autores.

Durante a cirurgia, anestesiaram-se os nervos alveolar superior anterior e nasopalatino. Na região foi executado um retalho mucoperiosteal com lâmina de bisturi ${ }^{\circ} 15$ para exposição da área apical do elemento 22 . A partir da fenestração já anteriormente presente na cortical vestibular, acessou-se a lesão. Em seguida, a lesão periapical foi retirada pela técnica de enucleação com Cureta de Lucas, apresentando aproximadamente 10mm (Figura 2).

Figura 2: Remoção da lesão cística.

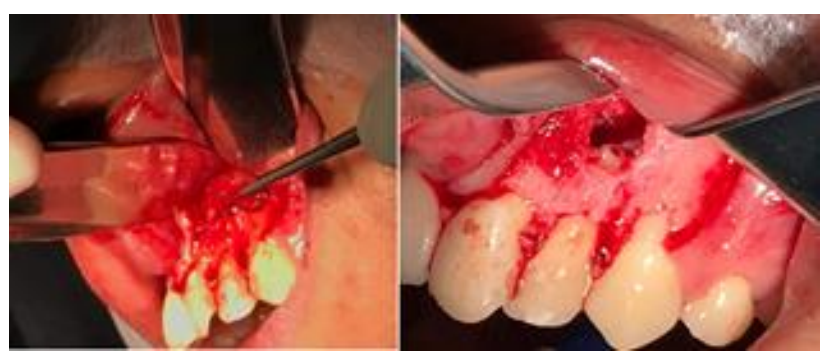

Fonte: Autores.

O material removido foi encaminhado posteriormente para um exame anatomopatológico comprovando que, de fato, tratava-se de um cisto periapical (Figura 3). Procedeu-se com a apicectomia do elemento e lavagem do sítio de exposição com soro fisiológico, finalizando com a secagem do local. 
Figura 3: Fotomicrografia da peça operatória.

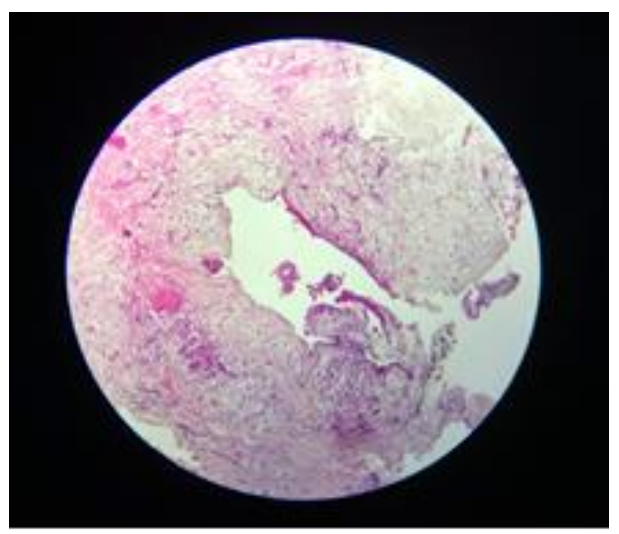

Fonte: Autores.

O ápice exposto foi irrigado com soro fisiológico, secando-se em seguida. De imediato, aplicou-se o Mineral Trióxido Agregado (MTA) como material retro-obturador (Figura 4). O retalho foi reposicionado e então o local foi suturado com fio não reabsorvível e a remoção da sutura foi agendada para a semana seguinte.

Figura 4: Obturação retrógrada com MTA.

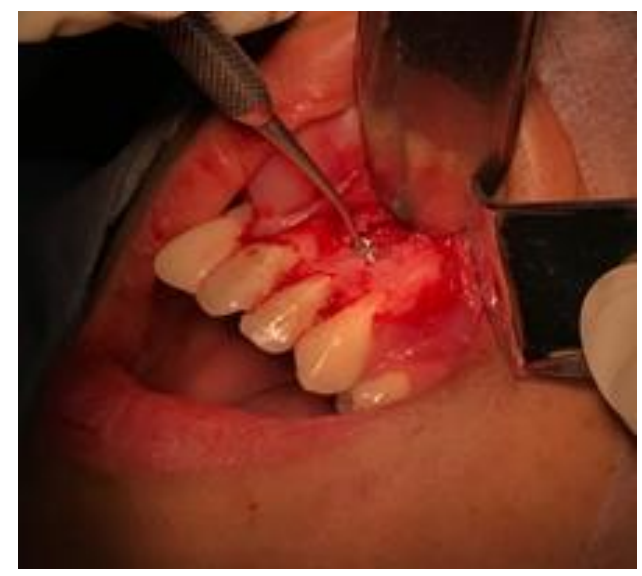

Fonte: Autores.

Prescreveu-se o uso de Amoxicilina 500mg de 12/12h durante sete dias, Nimesulida 100g de 12/12h por três dias e Dipirona 500mg a cada $6 \mathrm{~h}$ por 03 dias.

Após quinze dias de finalizada a cirurgia, o paciente retornou e percebeu-se, então, a ausência de drenagem da coleção purulenta e remissão da infecção, além de ausência de sintomatologia, evidenciando clinicamente a eficácia do procedimento realizado (Sousa et al., 2014). A obturação do sistema de canais foi realizada por meio de cones rolados associado ao cimento AH-Plus.

Realizou-se radiografia periapical da área após 6 meses da cirurgia, em que se observou remodelação óssea significativa no sítio da lesão (Figura 5). Após um ano, realizou-se a proservação clínica e radiográfica de 1 ano, que determinou o completo reparo da lesão periapical e ausência de sintomatologia dolorosa (Figura 6). 
Figura 5: Radiografia de proservação com 6 meses.

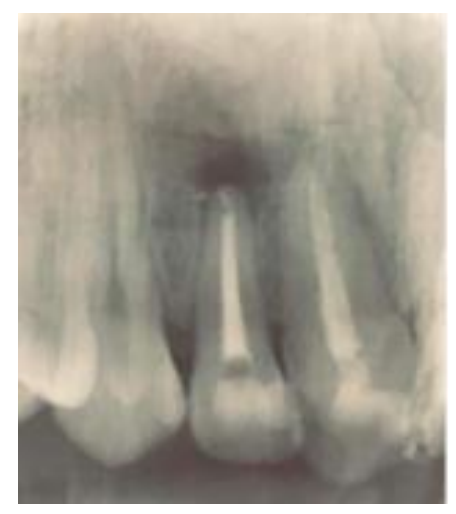

Fonte: Autores.

Figura 6: Radiografia final, 1 ano após a cirurgia.

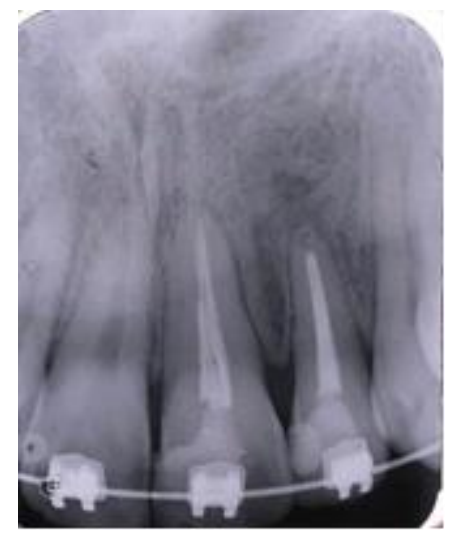

Fonte: Autores.

\section{Discussão}

Os cistos radiculares, em geral, apresentam-se como lesões silenciosas em região anterior da maxila, exceto quando associadas a algum foco de infecção. Nessas situações, atinge grandes extensões, em que se pode observar sinais clínicos de sua contaminação, como: edema, assimetria facial, dor espontânea e presença de exsudato purulento, resultando em drenagem espontânea via canal, como caracterizado no caso aqui ilustrado (Dantas et al., 2014; Vasconcelos et al., 2012).

De acordo com Pavelski et al., (2016), no tratamento endodôntico realizado primariamente, o especialista não está consciente do nível histológico de evolução da lesão periapical, o que levaria mais casos de insucesso quando presente. Como observado no presente caso, a eliminação da causa infecciosa pelo tratamento endodôntico primário não foi efetivo, e sabe-se que o tipo de lesão e o sucesso do tratamento estão fortemente associados.

Segundo Siqueira Jr. et al., (2012), a necropulpectomia seria o tratamento de primeira escolha para o caso referido, entretanto, devido às dificuldades de obturação via canal radicular, utilizou-se da enucleação via cirurgia parendodôntica para remover o cisto periapical. Sabe-se que a cirurgia está indicada em casos que o tratamento endodôntico prévio não foi suficiente para haver remissão dos sinais clínicos e radiográficos da lesão periapical. Ela objetiva à remoçãodo ápice radicular, erradicando a infecção presente nos tecidos radiculares e perirrradiculares. Justifica-se também a sua indicação juntamente com a obturação retrógrada com a finalidade de evitar corretamente o ápice e, assim, aumentar o índice de sucesso do tratamento. Tornou-se necessário, então, a intervenção multidisciplinar para solução do caso, integrando a endodontia com a cirurgia (Azambuja et al., 2006; Guimarães et al., 2006; De Miranda et al., 2018; Fagundes et al., 2011).

Além disso, o uso conjunto da apicectomia viabilizou a retrobturação, instrumentando o canal e reduzindo o número 
de infectantes advindos dos tecidos perirradiculares. A exposição do ápice, nesse caso, facilitou a instrumentação e a inserção do material obturador MTA, o qual, após 1 ano de procedimento, possibilitou neoformação óssea expressiva do conduto radicular.

Dessa forma, a exposição do segmento permite visão direta da região seccionada, facilitando uma obturação com um material biocompatível e, que, no caso abordado, foi fundamental para evitar o extravasamento de guta percha e cimento obturador (Oliveira et al., 2017; Fehlberg \& Bittencourt, 2019; Silva et al., 2021).

Um outro fator a ser levado em consideração é a necessidade de se ter um canal seco após a apicectomia e que possibilite a obturação retrógrada imediata, por conta disso, tomou-se os cuidados de lavagem com soro fisiológico e posterior secagem para que o MTA aderisse à superfície satisfatoriamente (Sette-Dias et al., 2010).

Ademais, o MTA é um material altamente indicado em casos de prognósticos desfavoráveis, pois aumenta as taxas de sucesso do tratamento. Ele age por meio do estímulo à reparação dos tecidos perirradiculares, ativando cementoblastos para produzir matriz de cemento pela presença de $\mathrm{pH}$ alcalino, resultando na deposição de cemento em casos de apicectomia. Possui capacidade de reduzir a inflamação local e apresenta, também, melhores resultados em relação a outros materiais retro obturadores quando observados ao microscópio óptico (Sousa et al., 2014; De Farias et al., 2006; Gomes et al., 2009; De Mello-Neto et al., 2010; Saunders, 2008).

\section{Considerações Finais}

Fica claro, portanto, que a cirurgia parendodôntica associada à apicectomia e obturação retrógrada com MTA, em situações de drenagem de coleção purulenta via canal, é uma excelente alternativa para casos de cisto periapical. A proservação clínica e radiográfica determinou o completo reparo da lesão periapical e ausência de sintomatologia dolorosa. Recomenda-se a utilização da técnica descrita neste estudo em casos semelhantes, de forma a otimizar o diagnóstico e a solução de

\section{Referências}

Almeida-Filho, J., Almeida G. M., Marques, E. F. \& Bramante C. M. (2011). Cirurgia Paraendodôntica: relato de caso. Revista Oral Sciences, 3(1), 21-25.

Azambuja, T. W. F., Bercini, F. \& Alano F. (2006). Cirurgia paraendodôntica: revisão da literatura e apresentação de casos clínico-cirúrgicos. Revista da Faculdade de Odontologia de Porto Alegre, 47(1), 24-29

Dantas R. N. X., Dultra, J. A., Borges, G.L., Dultra, F. K. A. A. \& Neri R. F. A. (2014). Enucleação de cisto radicular maxilar associado à apicectomia: relato de caso. Revista de Cirurgia e traumatologia Buco-maxilo-facial, 14(2), 21-26.

D’Azzio, P. S. S., Assis, N. M. S. P., Campos, C. N., Bastos, T. R. \& Lopes, R. M. (2010). O uso da tomografia cone beam no auxílio ao diagnóstico e planejamento de cirurgia periapical: relato de caso clínico. Odontologia Clínico-Científico, 9 (4), 377-380.

De Farias J. G., Rasquin L. C. \& Gonçalves A. P. R. (2006). Cirurgia paraendodôntica utilizando o MTA como material retrobturador: relato de casos. Revista de Cirurgia e traumatologia Buco-maxilo-facial, 6(4), 57-64.

De Mello Neto O. L., Souza A. S., Machado, M. E. L. \& Sydney, G. B. (2010). Capacidade de selamento marginal apical propiciado por alguns materiais retrobturadores. Revista Gaúcha de Odontologia, 58(4), 497-501.

De Miranda, P. B., Ferranti, K. N. \& Grando, C. P. (2018). Cirurgia parendodôntica associada a enxerto ósseo com biomaterial: relato de caso. PERSPECTIVA, Erechim, 42(157), 123-130.

De Oliveira, G. A. A., Silva, F. E., Alves, T. K. C., Rodrigues, E. S., de Aguiar M. A. \& Amorim, J. C. F. (2017). Cirurgia paraendodôntica: Apicetomia com curetagem periapical. Revista Arquivo Brasileiro de Odontologia, 13(1), 112-118.

Fagundes, R. B., Do Prado, M., Gomes, B. P. F. A., Damé, J. A. M. \& De Sousa, E. L. R. (2011). Cirurgia parendodôntica: uma opção para resolução de perfuração radicular - apresentação de caso clínico. Revista de Odontologia da UNESP, 40(5), 272-277.

Fehlberg, B. K. \& Bittencourt, G. (2019). Parendodontic surgery — apicoectomy andsimultaneous obturation of root canals with mineral trioxide aggregate (MTA): case report. Dental Press Endodontics, 9(1), 48-57.

Gomes, C. C., Acceta, R. F., Camões, I. C. G., Freitas, L. F. \& Pinto, S. S. (2009). Análise da Adaptação Marginal de Materiais Retrobturadores Análise da Adaptação Marginal de Materiais Retrobturadores. Pesquisa Brasileira em Odontopediatria e Clínica Integrada, 9(1), 31-35. 
Research, Society and Development, v. 11, n. 2, e29911225802, 2022

(CC BY 4.0) | ISSN 2525-3409 | DOI: http://dx.doi.org/10.33448/rsd-v11i2.25802

Graciano, N. R., Branco, G. S., Nascimento, V. R., Pfau, E. A., Brunini, S. H. S. \& Tomazinho, L. F. (2021). Cirurgia parendodônticado com retro-preparo e retro-obturação: relato de caso. Brazilian Journal of Surgery and Clinical Research - BJSCR, 34(1), 24-28.

Guimarães, K. B., Post, L. K., Bezerra, M. F., Isolan, C. P. \& Hosni, E. S. (2006). Cirurgia parendodôntica com obturação simultânea dos canais radiculares: relato de caso clínico. Revista de Ciências Médicas e Biológicas, 5(2), 188-194.

Pavelski, M. D., Portinho, D., Casagrande-Neto, A., Griza, G. L. \& Ribeiro, R. G. (2016). Paraendodontic surgery: case report. RGO, Revista Gaúcha de Odontologia, 64(4), 460-466.

Pereira A. S. et al. (2018). Metodologia da pesquisa científica. UFSM

Pozza, D. H., Moreira, C. C., Post, L. K., Xavier, C. B. \& De Oliveira, M. G. (2005). Avaliação de técnica cirúrgica parendodôntica: apicectomia em 90, retrocavitação com ultra-som e retrobturação com MTA. Revista Odonto Ciência - Faculdade de Odontologia/PUCRS, 20(50), 308-312.

Saunders, W. P. (2008). A Prospective Clinical Study of Periradicular Surgery Using Mineral Trioxide Aggregate as a Root-end Filling. Journal of Endodontics, 34(6), 660-665.

Sette-Dias, A. C., Maltos, K. L. M. \& De Aguiar E. G. (2010). Tratamento endodôntico transcirúrgico: uma opção para casos especiais. Revista de Cirurgia e traumatologia Buco-maxilo-facial, 10(2), 49-53.

Silva, J. S. \& Oliveira, R. V. (2016). Cirurgia para-endodôntica: relato de caso clínico. Revista UNINGÁ, 29(1), 103-106.

Silva, S. A., Laranjeira, A. C. S., Velozo, C., Montenegro, L. A. S, Bernardo, B. B. B., Santos, M. B. S., \& Albuquerque, D. S. (2021). Regeneraçãoapós cirurgia paraendodôntica em dente com extensa fenestração óssea-Relato de caso com acompanhamento de 3 anos. Research, Society and Development, 10(4), e22210413983.

Siqueira Júnior, J. F., Rôças, I. N., Lopes, H. P., Alves, F. R.F., Oliveira, J. C. M., Armada, L. \& Provenzano, J. C. (2012). Princípios biológicos do tratamento endodôntico de dentes com polpa necrosada e lesão perirradicular. Revista Brasileira de Odontologia, 69(1), 8-14.

Sousa, N. B., Nunes, M. A. C., Veloso, K. M. M. \& Pereira, A. F. V. (2014). Agregado de trióxido mineral e uso como material retro-obturador em cirurgia paraendodôntica. Revista Brasileira de Odontologia, 71(2), 144-147.

Travassos, R. M. C., Negreiros, J. H. C. N., Farias, W. D. S., Soares, T. B. P., Barbosa, L. M., Souza, T. G. S., \& Silva, H. J. (2020). Apicectomia e obturação retrógrada de dente com calcificação radicular interna: relato de caso. Research, Society and Development, 9(9), e327997390.

Vasconcelos, R. G., Queiroz, L. M. G., Alves Júnior, L. C., Germano, A. R. \& Vasconcelos, M. G (2012). Abordagem Terapêutica em Cisto Radicular de Grandes Proporções - Relato de Caso. Revista Brasileira de Ciências da Saúde, 16(3), 467-474. 